\title{
Endoscope-Assisted Retrosigmoid Approach for Cerebellopontine Angle Epidermoid Tumor
}

\author{
Francisco Vaz-Guimaraes ${ }^{1}$ Paul A. Gardner ${ }^{2}$ Juan C. Fernandez-Miranda ${ }^{2}$ \\ ${ }^{1}$ Department of Neurosurgery, Baylor College of Medicine, Houston, \\ Texas, United States \\ 2 Department of Neurological Surgery, University of Pittsburgh \\ Medical Center, Pittsburgh, Pennsylvania, United States \\ Address for correspondence Juan C. Fernandez-Miranda, MD, \\ Department of Neurological Surgery, Stanford University, 875 Blake \\ Wilbur Drive, CC2222, Stanford, CA, 94305, United States \\ (e-mail: drjfm@stanford.edu).
}

J Neurol Surg B 2018;79(suppl S5):S409-S410.

\begin{abstract}
Keywords

- cerebellopontine angle

- endoscope-assisted

- epidermoid tumor

- retrosigmoid approach

Objectives Surgical resection is the only effective treatment modality for epidermoid tumors. Complete resection with preservation of neurological function must be pursued whenever possible, because it offers a cure for patients. However, the inability to identify hidden remnants, interdigitating around cranial nerves, especially in larger tumors, may be a contributing factor for incomplete resection. This operative video demonstrates the technical nuances in achieving complete resection of a cerebellopontine angle epidermoid tumor via an endoscope-assisted retrosigmoid approach. Design and Setting Operative video of an endoscope-assisted retrosigmoid, approach for complete resection of a cerebellopontine angle epidermoid tumor. The patient was a 16-year-old female, who presented with 1-year history of worsening headaches and imbalance. Her neurological exam was normal, including normal cranial nerve function, and hearing. Radiological evaluation revealed an epidermoid tumor in the right cerebellopontine angle, extending to the interpeduncular cistern. Surgical resection was recommended. Given extension of the tumor across the midline, an endoscope-assisted procedure was planned to increase the odds of complete resection. Results The video demonstrates the surgical technique applied for tumor resection. The patient's clinical symptoms resolved completely after surgery and she remained neurologically intact. Postoperative magnetic resonance imaging (MRI) confirmed complete tumor resection. There were no postoperative complications.

Conclusions The use of endoscopic techniques for resection of cerebellopontine angle epidermoid tumor is safe and effective and may increase the odds of complete resection, especially in larger tumors spreading across the midline, by enabling the surgeon clear visualization of deep-seated and contralateral relevant neurovascular structures, not readily accessible by the surgical microscope.

The link to the video can be found at: https://youtu.be/X6YP_7OeQQE.
\end{abstract}

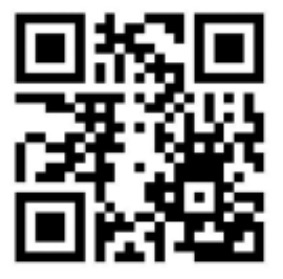

received

June 1, 2018

accepted

August 11, 2018

published online

October 16, 2018 www.thieme.com/skullbasevideos

www.thieme.com/jnlsbvideos

DOI https://doi.org/

10.1055/s-0038-1669983. ISSN 2193-6331.
๑) 2018 Georg Thieme Verlag KG
Stuttgart · New York

License terms

(c) $(1) \$$ 


\section{Note}

This work has not been presented at any medical conference.

\section{Conflict of Interest}

All authors certify that they have NO affiliations or involvement with any organization or entity with any financial interest (such as honoraria; educational grants; participation in speaker's bureau; membership, employment, consultancies, stock ownership, or other equity interest; and expert testimony of patent-licensing arrangements), or nonfinancial interest (such as, personal or professional relationships, affiliations, knowledge, or beliefs) in the subject matter or materials discusses in this manuscript.

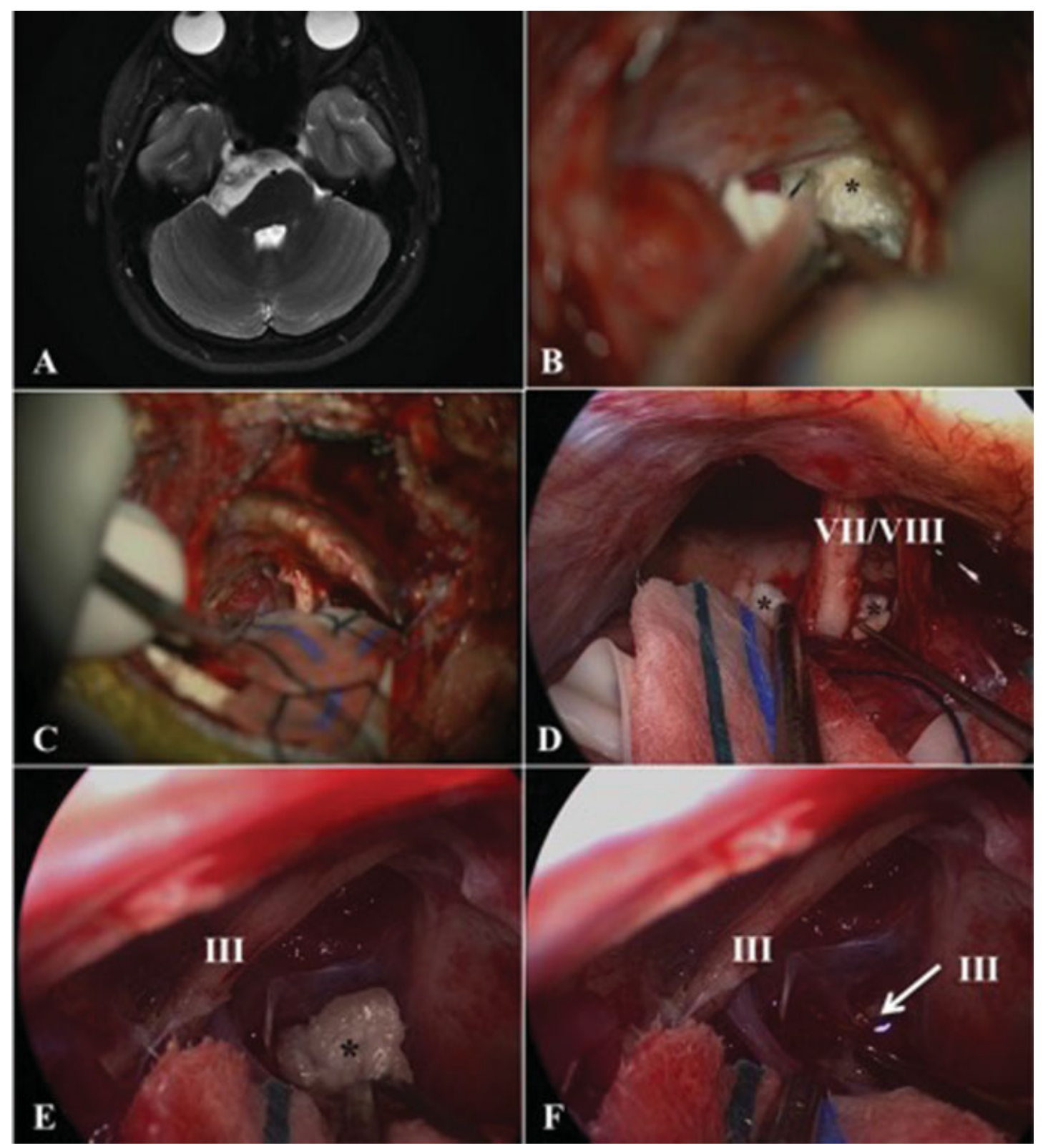

Fig. 1 (A) preoperative, T2-weight axial MRI showing a right CPA epidermoid cyst; (B) Microscopic view at the beginning of the operation after retromastoid craniectomy; (C) Microscopic view after the microscopic resection of the cyst; (D) Endoscopic view (0-degree endoscope) of the CPA. Note the now visible residuals attached to the origin of the trigeminal (V), facial (VII) and vestibulocochlear (VIII) nerves; (E and F) Endoscopic view (0-degree endoscope) and identification of hidden remnants close to the left oculomotor (III) nerve exit zone. MRI, magnetic resonance imaging, CPA, cerebellopontine angle, ${ }^{*}$ cyst. 